\title{
Reliable and Precise Neuronal Firing during Sensory Plasticity in Superficial Layers of Primary Somatosensory Cortex
}

\author{
Brett L. Benedetti, ${ }^{1,2}$ Stanislaw Glazewski, ${ }^{3}$ and Alison L. Barth ${ }^{1,2}$ \\ ${ }^{1}$ Department of Biological Sciences and ${ }^{2}$ Center for the Neural Basis of Cognition, Carnegie Mellon University, Pittsburgh, Pennsylvania 15213, \\ and ${ }^{3}$ School of Life Sciences, Institute for Science and Technology in Medicine, Keele University, Staffordshire, Keele, ST5 5BG, United Kingdom
}

\begin{abstract}
Neocortical neurons show astonishing variation in the presence and timing of action potentials across stimulus trials, a phenomenon whose function and significance has been the subject of great interest. Here we present data showing that this response variability can be significantly reduced by altered sensory experience. Removal of all but one whisker from the side of the mouse face results in the rapid (within $24 \mathrm{~h}$ ) potentiation of mean firing rates within the cortical representation of the spared whisker in young postnatal animals (postnatal days 13-16). Analysis of single-unit responses from whisker-spared animals shows that this potentiation can be attributed to an enhancement of trial-to-trial reliability (i.e., reduced response failures), as well as an increase in the mean number of spikes evoked within a successful trial. Changes were confined to superficial layers $2 / 3$ and were not observed in the input layer of the cortex, layer 4 . In addition to these changes in firing rates, we also observed profound changes in the precise timing of sensory-evoked responses. Trial-totrial temporal precision was enhanced and the absolute latency of responses was reduced after single-whisker experience. Enhanced spike-timing precision and trial-to-trial reliability could also be triggered in adolescent animals with longer periods (7 d) of singlewhisker experience. These experiments provide a quantitative analysis of how sensory experience can enhance both reliability and temporal precision in neocortical neurons and provide a framework for testing specific hypotheses about the role of response variability in cortical function and the molecular mechanisms underlying this phenomenon.
\end{abstract}

\section{Introduction}

Individual neurons within the neocortex appear to be strikingly inefficient at transmitting information, evidenced by a pronounced trial-to-trial variability in stimulus-driven firing (Shadlen and Newsome, 1998). Despite excellent stimulus-response reliability in the periphery and subcortical areas (Glazewski et al., 1998; Kara et al., 2000; Brecht and Sakmann, 2002; Reinagel and Reid, 2002; Jones et al., 2004; Arabzadeh et al., 2005; de Kock et al., 2007), neurons in primary sensory cortex display substantial variability in response to the same, repeated stimulus (Mainen and Sejnowski, 1995; Reinagel and Reid, 2000; Brecht and Sakmann, 2002; de Kock et al., 2007). Indeed, even when stimulus-driven firing in a neuron can be reliably elicited in vivo, the timing of this evoked response can be highly imprecise (de Kock et al., 2007). It has been hypothesized that the CNS may tolerate this extraordinary variability by using redundant neural coding strategies, in which the firing of only a small subset of cells may be sufficient to encode a stimulus (Houweling and Brecht, 2008; Huber et al., 2008). Alternatively, response variability in an individual neuron

\footnotetext{
Received July 16, 2009; accepted Aug. 10, 2009.

This work was supported by National Institutes of Health Grant DA017188-01 (A.L.B.) and the Physiological Society (United Kingdom) (S.G.). We thank members of the Barth and Urban laboratories for helpful comments on this manuscript.

Correspondence should be addressed to Alison L. Barth, Box 142, Mellon Institute, Carnegie Mellon University, 4400 Fifth Avenue, Pittsburgh, PA 15213. E-mail: barth@cmu.edu.

D0I:10.1523/JNEUROSCI.3431-09.2009

Copyright $\odot 2009$ Society for Neuroscience $\quad$ 0270-6474/09/2911817-11\$15.00/0
}

may be a measure of uncertainty about stimulus characteristics, a feature that may allow better estimation of risk in determining behavioral response (Ma et al., 2006).

Previous work has established that whisker-driven sensory activity can trigger overall potentiation of evoked firing rates in neocortical neurons (Diamond et al., 1994; Glazewski and Fox, 1996; Barth et al., 2000; Glazewski et al., 2007). Analysis in previous studies has typically focused on mean firing rates, averaged over multiple trials. Remarkably, the detailed basis for the increase in mean firing rate-for example, an increase in the number of evoked spike per successful trial, a reduction in trial-to-trial failures, or an increase in the timing precision of spikes- has not been systematically evaluated. To develop and test specific hypotheses about the cellular and molecular mechanisms involved in experience-dependent plasticity, it is essential to document precisely what type of changes in response properties actually occur in vivo.

Experience-driven plasticity in the barrel cortex affords an excellent opportunity to understand whether improvements in spike-timing precision and/or response reliability are engaged to increase stimulus-driven spike output. Enhanced timing precision and trial-to-trial reliability of neuronal firing will increase the information carried by a cell, facilitating temporal summation of inputs to downstream neurons that can effectively drive recurrent activity through the network (Oviedo and Reyes, 2002; Bruno and Sakmann, 2006; Sarid et al., 2007). The present study is primarily focused on young postnatal animals, at a time at 
which neuronal firing responses undergo rapid maturation (Stern et al., 2001).

Our results show that that experiencedependent plasticity in primary somatosensory (barrel) cortex occurs through increased response reliability and is concurrent with enhanced spike time precision. Removal of all but a single whisker from both sides of the mouse face [singlewhisker experience (SWE)] in young postnatal animals results in a significant and rapid potentiation of evoked firing rates in layer $2 / 3$, but not layer 4 , in the cortical representation of the spared whisker. Before single-whisker sparing, whisker deflection evokes unreliable firing of layer $2 / 3$ neurons, and evoked spikes occur at variable latencies, across trials within the same cell. After $24 \mathrm{~h}$ of SWE, mean firing rates nearly double, and trialto-trial response reliability was increased almost twofold. Evoked spikes occurred earlier with respect to the stimulus, and the SD of spike latencies, a measure of spike-timing precision or "jitter," was significantly reduced. These parameters were similarly altered in 5- to 8-week-old animals, but required significantly longer periods of SWE to become apparent. Thus, we find that high variability in neuronal response properties is not a fixed and necessary feature of neocortical circuits but rather can be dynamically modulated by experience.

\section{Materials and Methods}

Animals. Recordings were made from 217 neurons in 26 wild-type C57BL/6 mice aged postnatal day 13 (P13) to P16 on the day of recording and 64 neurons in 6 C57BL/ 6 mice aged 5-8 weeks. New analysis was also performed on data from an additional 197 neurons from 13 animals collected in a previous study (Glazewski et al., 2007). All experiments were performed with the approval of the Carnegie Mellon University Institutional Animal Care and Use Committee.

Deprivation. Animals (young postnatal; aged P12-P15 or adolescent; aged 5-8 weeks on the day of plucking) were subjected to $24 \mathrm{~h}$ of SWE in which all large facial vibrissae were removed from both sides of the snout except for the D1 whisker on one side. Note that this treatment is different than we have used previously in adolescent animals (Barth et al., 2000). Vibrissae were removed by plucking under light isoflurane anesthetic. Control animals underwent a sham-plucking procedure in which they were anesthetized and handled just as their deprived littermates. Animals were returned to their home cages for $24 \mathrm{~h}$ before electrophysiological recording (P13-P16 for young postnatal animals). Seven days of SWE was followed by 7-9 d of whisker regrowth, as described previously (Glazewski et al., 2007).

Anesthesia and surgery. On the day of recording, animals were anesthetized with urethane $(1.0-1.5 \mathrm{~g} / \mathrm{kg}$ body weight with trace amounts of acepromazine intraperitoneally; Sigma). Depth of anesthesia was monitored by the absence of hindlimb withdrawal after pinch and corneal blink reflexes. Supplemental doses of urethane (10\% of initial dose) were administered to maintain anesthesia depth. Body temperature was maintained at $37^{\circ} \mathrm{C}$ using a rectal thermometer and heating blanket (Harvard Apparatus). Animals were restrained in a stereotaxic instrument (David Kopf Instruments), and the skull overlying the barrel cortex was thinned using a dental drill (FHC). For each electrode penetration, a small hole was made in the thinned skull using a 30 gauge hypodermic needle tip. The skull was kept moist by superfusion of $0.1 \mathrm{M}$ phosphate buffer (PB).

Recording. Extracellular single-unit recordings were made in layers $2 / 3$ and $4(0-450 \mu \mathrm{m})$ of the barrel cortex using glass-insulated carbon-fiber electrodes as described previously (Glazewski et al., 2007). Signals were amplified, filtered, and digitized at $20 \mathrm{kHz}$ (CED Micro1401; Cambridge Electronic Design). Single units were isolated online using a voltage window discriminator (Digitimer). Event outputs were digitized and acquired using Spike2 software.

Electrode penetrations were made in the spared-barrel column (D1 barrel column) of SWE mice and in D1 and surrounding barrel columns of control mice. Whisker deflections $\left(1^{\circ}\right.$ vertical displacement, $10 \mathrm{~ms}$ duration using a glass pipette placed $10 \mathrm{~mm}$ from the follicle) were delivered by driving a piezoelectric bimorph wafer with a voltage source (Digitimer) triggered by Spike2 software. For each unit, 50 deflections were delivered to the principal whisker $(\mathrm{PW})$ at a frequency of $1 \mathrm{~Hz}$. The PW was determined experimentally as the whisker that elicited the largest short-latency response in layer 4 and verified histologically post hoc (see below). Spontaneous unit activity, in which no whiskers were stimulated, was subsequently collected (50 s).

Histology. After recording in each penetration, a small electrolytic lesion $(1.1 \mu \mathrm{A}$ for $10 \mathrm{~s})$ was made in layer 4 to mark the recording location. At the end of each experiment, animals were deeply anesthetized with urethane and transcardially perfused with $0.1 \mathrm{M}$ phosphate buffer, followed by $4 \%$ paraformaldehyde in $0.1 \mathrm{~m}$ phosphate buffer (PFA). The brain was removed, and the cortex was then flattened and fixed in PFA/ $20 \%$ sucrose mixture overnight. Tissue was then sunk in $30 \%$ sucrose $/ 0.1$ M $\mathrm{PB}$, sectioned to $40 \mu \mathrm{m}$ thickness on a freezing microtome, and processed for cytochrome oxidase reactivity to identify barrel location. Images of stained sections were analyzed to confirm all recording locations and electrode depths. For animals undergoing SWE deprivation, only penetrations located in the D1 barrel column were included in the analysis (Fig. 1). We found the border between layer $2 / 3$ and layer 4 to be at $300 \mu \mathrm{m}$; therefore, cells between 0 and $300 \mu \mathrm{m}$ below the pial surface were classified as belonging to layer $2 / 3$, and cells between 301 and 450 $\mu \mathrm{m}$ were classified as layer 4 .

Data analysis. Poststimulus time histograms (PSTHs) were constructed offline for each unit using Spike2 software. Within each group (layer 4, control or SWE, and layer 2/3, control or SWE), the number of spikes in each bin was averaged over all cells to create a population PSTH. Because evoked activity was heavily concentrated between 0 and $55 \mathrm{~ms}$ after stimulus, with some recurrent activity persisting to $\sim 200 \mathrm{~ms}$ after stimulus before firing rates returned to baseline spontaneous firing levels (supplemental Fig. 2, available at www.jneurosci.org as supplemental material), response magnitudes [spikes per stimulus (s/s)] were determined as the number of spikes between 0 and 55 ms elicited per whisker deflection (average of 50 stimulus trials). Spikes occurring at very short 
latencies that cannot be related to whisker stimulation $(0-3 \mathrm{~ms})$ were excluded.

To quantify trial-to-trial response reliability, we developed a reliability index for each cell by calculating the fraction of whisker stimulation trials (50 total) in which the cell fired at least one spike within the analysis window described above. Thus, a reliability index value of 0 indicates no action potential (AP) firing during any trials, a value of 0.5 indicates firing on 25 of the trials, and a value of 1 indicates that the cell fired at least one AP on every stimulus trial. In addition, the mean number of spikes per successful trial was also calculated for each cell.

The temporal precision of evoked responses was quantified by calculating the latency of the first spike evoked by whisker stimulation in each trial. Stimulus trials resulting in no spikes were not included in the latency analysis. For each cell, all first-spike latencies were averaged to generate a mean latency. Furthermore, the SD of these spikes was calculated to measure the temporal precision (jitter) of these responses. Note that, for both latency and precision calculations, cells were excluded from the population analysis if whisker stimulation only evoked a spike on a single trial (because this would give an SD of 0 ).

Spontaneous activity was determined from data collection periods in which no whisker deflection was delivered and calculated as the mean firing rate over $50 \mathrm{~s}$ of recording.

Averaged data reflect the mean of all cells within a group (control layer $2 / 3$, control layer 4 , SWE layer $2 / 3$, and SWE layer 4 ). Normality was assessed for each dataset with the Shapiro-Wilks test. Because most distributions were not found to be normal, nonparametric statistical analyses were used for comparisons and implemented using R software ( R version 2.4.1). Groups were initially compared using the Kruskall-Wallis test. Pairwise comparisons were then performed using the Mann-Whitney $U$ test for group means and Kolmogorov-Smirnov test for distributions. Correlations were assessed using Spearman's test statistic $(\rho)$, and the corresponding $p$ values indicate significance of the test $(\rho=0$, i.e., test of no correlation). Data are shown as mean \pm SEM unless otherwise noted.

\section{Results \\ Single-whisker experience enhances stimulus-evoked firing rates}

Whisker-driven sensory experience can enhance the strength of glutamatergic synapses in young postnatal barrel cortex (Takahashi et al., 2003; Clem and Barth, 2006). However, altered sensory experience has also been shown to increase AP threshold in SWEdeprived mice (Barth et al., 2004). To determine whether an increase in synaptic strength is correlated with an overall potentiation of stimulus-driven firing rates at this age, in vivo singleunit recording experiments (Fig. $2 A, B$ ) were performed. We found that SWE was remarkably effective at driving rapid increases in the firing rate of spared-whisker-evoked responses in P13-P16 animals, similar to what has been described in older mice with longer periods of sensory experience (Diamond et al., 1994; Glazewski and Fox, 1996; Glazewski et al., 2007). After 24 h of SWE, the mean firing rate of neurons in layer $2 / 3$ of the spared barrel column nearly doubled compared with principal-whiskerevoked responses in control mice (control, $0.45 \pm 0.05 \mathrm{~s} / \mathrm{s}, n=82$ cells vs SWE, $0.84 \pm 0.09 \mathrm{~s} / \mathrm{s}, n=65$ cells; $p<10^{-5}$ ) (Fig. $2 D, E$ ) (supplemental Fig. $1 \mathrm{~A}$, available at www.jneurosci.org as supplemental material). In agreement with what has been found in older animals, mean firing rates in layer 4 were not altered by SWE in young postnatal animals (control, $1.02 \pm 0.1 \mathrm{~s} / \mathrm{s}, n=36$ vs SWE $0.90 \pm 0.09 \mathrm{~s} / \mathrm{s}, n=34 ; p=0.41$ ). Thus, the increased firing in layer $2 / 3$ cannot be directly attributed to increased activity in either subcortical areas or thalamo-recipient layer 4.

A comparison of evoked firing between layer L4 and layer $2 / 3$ neurons in control animals shows that firing rates in layer 4 are significantly higher than in layer $2 / 3$ (supplemental Fig. $1 A, B$, available at www.jneurosci.org as supplemental material), sug- gesting that activation of layer 4 and layer $2 / 3$ inputs is not typically sufficient to drive APs in layer 2/3 neurons. However, after SWE, evoked firing rates between layer 4 and layer $2 / 3$ neurons become statistically indistinguishable (supplemental Fig. $1 C$, available at www.jneurosci.org as supplemental material), indicating that, on a trial-to-trial basis, firing of layer 4 neurons now effectively drives firing in layer $2 / 3$ neurons. This is most apparent in Figure $2 F$, where the firing rate in control layer 4 neurons is greater than that observed in layer $2 / 3$ neurons across all poststimulus time bins. After SWE, the majority of evoked firing in layer 4 is concentrated within the short time interval $(0-16 \mathrm{~ms})$ immediately after the whisker deflection stimulus, but layer $2 / 3$ firing is delayed from that observed in layer 4 (18-28 ms) (Fig. 2).

\section{Single-whisker experience increases trail-to-trial reliability}

In theory, multiple factors may contribute to the increased firing rates observed during experience-dependent plasticity. Sensoryevoked responses are often unreliable, such that layer $2 / 3$ neurons typically respond to only a fraction of stimulation trials; thus, one way to increase firing rates would be to increase trial-to-trial response reliability. Alternatively, firing rates might go up because neurons fire more spikes per trial without changing the total number of responsive trials. Both mechanisms may act together to increase stimulus-evoked responses.

To evaluate these hypotheses, we began by analyzing SWEinduced changes in response reliability. For each cell, 50 stimulus trials were performed, and the presence of a spike within the first $55 \mathrm{~ms}$ was assessed (on average, most of the whisker-evoked activity was confined to this window) (supplemental Fig. 2, available at www.jneurosci.org as supplemental material). The example cell shown in Figure $3 A$ illustrates the high trial-to-trial response failures characteristic of layer $2 / 3$ neurons in control mice, where gray regions indicate trials with no response. A reliability index was calculated for each cell as the fraction of trials that evoked a response. After $24 \mathrm{~h}$ of SWE (for an example cell, see Fig. $3 B$ ), layer $2 / 3$ response reliability was significantly increased compared with control (control, $0.30 \pm 0.04, n=82$ cells vs SWE, $0.48 \pm 0.03, n=65$ cells; $p<10^{-5}$ ) (Fig. 3C). On average, the number of successful trials increased from 15 of 50 in control to 24 of 50 after SWE. Fully three-quarters of layer $2 / 3$ neurons in control animals exhibited low reliability $(\leq 50 \%)$. After SWE animals, only half the neurons showed this unreliable response (Fig. $3 D)(p<0.001)$.

In contrast, SWE failed to enhance the reliability of layer 4 responses above control levels (control, $0.60 \pm 0.04, n=36$ cells vs SWE, $0.56 \pm 0.03, n=34$ cells; $p=0.42$ ), indicating that the increased reliability in layer $2 / 3$ is not the direct result of more reliable evoked firing in layer 4.

Does SWE also trigger layer $2 / 3$ neurons to fire more spikes on a single trial? By analyzing only those trials in which layer $2 / 3$ neurons responded to whisker deflection, we were able to normalize responses to the above-mentioned changes in reliability. Whereas control layer $2 / 3$ neurons showed an average of $1.39 \pm$ 0.04 spikes per successful trial ( $n=82$ cells), SWE layer $2 / 3$ neurons exhibit a modest but significant potentiation of evoked firing (SWE, $1.56 \pm 0.06$ spikes per successful trial; $n=65$ cells; $p=$ $0.03)$ (Fig. 3E). This change could be attributed to an increase in the percentage of successful trials evoking more than one spike ( $26 \%$ control vs $37 \%$ SWE; $p=0.0014$; data not shown). In layer 4 , neither overall firing rates on successful trials (control, $1.58 \pm$ 0.07 spikes per successful trial, $n=36$ vs SWE, $1.52 \pm 0.06$ spikes per successful trial, $n=34 ; p>0.5$ ) (Fig. $3 E$ ) nor the percentage of trials evoking more than one spike (38\% control vs $35 \%$ 
SWE; $p>0.5$; data not shown) were altered. Thus, both increased firing on successful trials and increased trial-to-trial response reliability contribute to experiencedependent potentiation in layer $2 / 3$.

\section{Absolute evoked spike latencies are shorter after SWE}

Because the relative timing of APs within a cortical layer can significantly influence signal summation and propagation of activity across the network, an increase in the temporal coherence of short-latency spikes may enhance detection thresholds, allowing animals to discern stimulus parameters over fewer whisking cycles. To determine whether experience-dependent plasticity might alter the trial-to-trial precision of spike times, we analyzed the latency of the first spike evoked by whisker deflection (Fig. 4A,B). After SWE, a highly significant reduction in the mean latency of the initial stimulus-evoked AP was observed in layer $2 / 3$ neurons (control, $24.1 \pm 0.75 \mathrm{~ms}, n=76$ cells vs SWE, $19.6 \pm 0.5 \mathrm{~ms}, n=64$ cells; $p<10^{-6}$ ) (Fig. $4 D$ ). Importantly, this reduction in latency was not attributable to earlier spiking in layer 4 neurons (Fig. $4 C, D$ ), because mean latency was not altered by SWE in this layer (control, $19.1 \pm 0.72$ ms, $n=36$ cells vs SWE, $17.2 \pm 0.66, n=$ 33 cells; $p=0.21$ ), indicating that the locus for this effect is not likely to be in subcortical regions or in layer 4 .

For cells from both control and SWE conditions, we found a significant correlation between reliability and onset latency (Fig. $4 F$ ), such that the most reliable cells also responded earlier than less reliable ones. SWE strengthened this relationship (correlation coefficient, control, $-0.268, n=76$ cells, $p=0.019$ vs SWE, $-0.419, n=64$ cells, $p<0.001$ ), raising the possibility that the cellular and molecular mechanism underlying improvements in spike-timing and trial-to-trial response reliability proceed in parallel.

\section{Stimulus-evoked spikes are more precisely timed}

The relative timing of APs across cells in layer $2 / 3$ is thus an important indicator of how activity can be propagated across the network. In conjunction with the SWE-driven decrease in spike latency observed in layer $2 / 3$ cells, we also find that the distribution of latencies within neurons recorded from SWE-treated animals becomes more narrow (Fig. $4 E$ ). This has the effect of sharpening the population response around a single latency compared with the relatively wide distribution of latencies observed in the control population. This result is especially notable because it is well appreciated that evoked responses, even for neurons within the same cortical column, can vary greatly between cells (Kerr et al., 2007), and thus mean firing rates across single-units show a wide variance (supplemental Fig. 1, available at www. jneurosci.org as supplemental material).
Given this apparent increase in the temporal precision of the population response after SWE, we sought to determine whether such changes may occur at the single-cell level on a trial-by-trial basis. Example spike rasters from individual neurons are shown in Figure 5 for control and SWE groups. Layer 2/3 neurons showed reduced trial-to-trial variability in the timing of the first evoked spikes after SWE (Fig. 5A-C). The clustering of these initial spikes is apparent across SWE cells, all of which show enhanced temporal precision (i.e., smaller SD of spike times) compared with control cells matched for reliability (Fig. 5F) $(p=0.00070)$.

For each layer $2 / 3$ cell that was responsive on at least two stimulus trials ( 76 of 82 cells for control; 64 of 65 cells for SWE), we calculated the SD of timing of the first spike as a measure of trial-to-trial temporal precision (in which a small SD is indicative of high precision and large SD of low precision). Averaged over all cells, this precision was significantly enhanced after $24 \mathrm{~h}$ of SWE (control, $8.88 \pm 0.41 \mathrm{~ms}, n=76$ cells vs SWE, $6.9 \pm 0.45 \mathrm{~ms}, n=$ 64 cells; $p=0.0013$ ) (Fig. $5 G$ ). This reduction in jitter is not likely to be attributable to a more synchronous onset of activity in layer 4 , because the latency and precision of initial spike time was not altered by SWE in layer 4 (Fig. $5 G)(p=0.93)$. For both control 

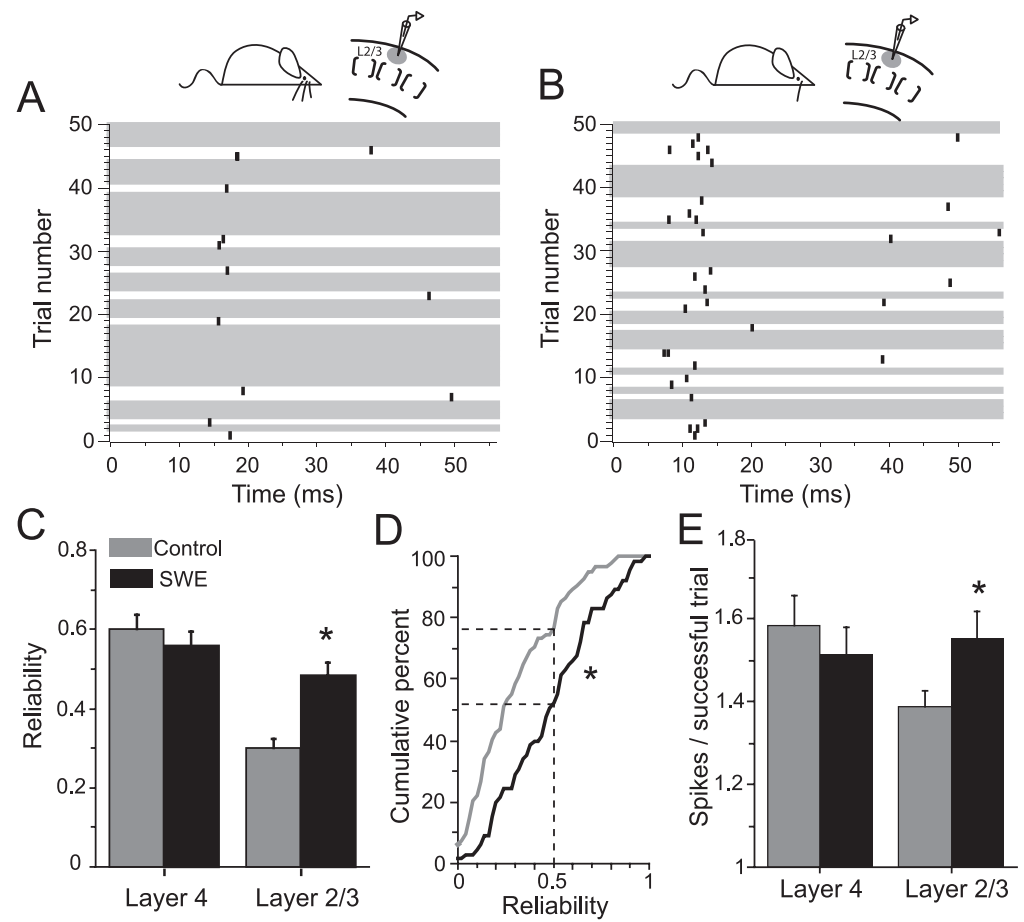

Figure 3. Enhanced trial-to-trial reliability contributes to plasticity in superficial layers. $A$, Raster plot of an example layer $2 / 3$ cell from a control mouse. Gray bars indicate trials in which whisker deflection failed to elicit an AP within the $55 \mathrm{~ms}$ analysis window. $\boldsymbol{B}$, Example raster plot after $S W E$. Note the more reliable response (i.e., fewer failures) compared with the cell in $\boldsymbol{A}$ (control reliability, 0.24; SWE, 0.54). C, Mean \pm SEM response reliability calculated for layer 4 and $2 / 3$ cells from control (gray) and SWE (black). ${ }^{*} p<10^{-4}$. D, Cumulative distribution of layer $2 / 3$ response reliabilities. ${ }^{*} p<10^{-4}$. Dashed lines indicate the percentage of cells in each population with reliabilities $<0.5$. E, Mean \pm SEM layer 4 and $2 / 3$ response magnitudes per successful trial. ${ }^{*} p<0.05$.

and SWE conditions, layer 2/3 neurons with the shortest latency responses showed a tendency to have the most precise spike times across the 50-stimulus trials (Fig. 5D). Furthermore, in control cells, we found a significant correlation between the precision and reliability of the response of each cell (Fig. $5 E$ ) (Spearman's correlation coefficient, $\rho=-0.25, p=0.028)$. However, no such relationship was observed after SWE $(\rho=-0.05, p=0.70)$, indicating that spike-timing precision was equally enhanced by experience-dependent plasticity in both reliable and unreliable cells.

\section{Potentiation of recurrent activity in layer $2 / 3$}

The dense interconnectivity of layer $2 / 3$ pyramidal neurons (Feldmeyer et al., 2006; Cheetham et al., 2007; Lefort et al., 2009) suggests that improved trial-to-trial reliability and timing precision across the population might to lead to increased recurrent activity within the network. To evaluate this, we analyzed delayed firing in two $150 \mathrm{~ms}$ windows after the stimulus (55-205 and 205-355 ms) (Fig. 6 A, B) (supplemental Fig. 2 F, available at www. jneurosci.org as supplemental material). At longer latencies (55$205 \mathrm{~ms}$ ) after whisker deflection, the probability that there was any spiking activity within a given trial was slightly enhanced after SWE compared with control (control, $16.9 \pm 1.5 \%, n=82$ cells vs SWE, $22.4 \pm 1.8 \%$ of trials, $n=65$ cells; $p=0.012$ ) (Fig. $6 C$ ). Enhanced activity within this temporal window is likely to be stimulus evoked for two reasons. First, spontaneous firing activity, assessed over a $50 \mathrm{~s}$ window in which no stimulus was delivered, was not altered between conditions (control, $0.75 \pm 0.06$ $\mathrm{Hz}, n=82$ cells vs SWE, $0.85 \pm 0.07 \mathrm{~Hz}, n=65$ cells; $p=0.17$ ) (Fig. $6 D$ ). In addition, there was no difference in the probability that there was any spiking activity in a given trial, measured in the 205-355 ms window (control, $9.0 \pm 0.08 \%$ trials, $n=$ 82 cells vs SWE, $9.4 \pm 1.2 \%, n=65$ cells; $p=0.79$ ) (Fig. 6C), suggesting that responses have returned to basal levels by this time (supplemental Fig. 2, available at www.jneurosci.org as supplemental material). Neither longer-latency firing nor spontaneous firing activity was significantly different in layer 4 across conditions (data not shown).

\section{Firing rate potentiation and enhanced temporal precision can be dissociated} Are the changes in the timing of neuronal firing induced during SWE dependent on the observed increases in firing rate and response reliability? A stronger postsynaptic potential (PSP) evoked by whisker deflection is one explanation for the increased firing rates induced after SWE, and increased PSP amplitude can enhance spike-timing precision, at least in vitro (Daw et al., 2006). Therefore, neurons in which firing rates have been potentiated may inevitably exhibit reduced onset latencies and higher temporal precision. Alternatively, SWE may activate distinct molecular pathways that independently alter response magnitude and response timing.

To examine whether these properties could occur independently of each other-in other words, whether enhanced temporal precision and reduced spike latency could be observed in the absence of firing rate potentiation-we analyzed data from layer $2 / 3$ SWE neurons with low firing rates that were comparable with values from control animals (Fig. 7A). Neurons from control and SWE mice with firing rates $>0$ but less than or equal to 0.5 were selected for analysis. If short-latency, precise spikes are only observed in neurons with high firing rates, then this select group should not exhibit changes in either property. An important caveat here is that, because it was not possible to record from the same neurons before and after SWE, this group of neurons may actually show increased stimulus-evoked firing compared with their own baseline control values. Thus, we cannot be certain that PSPs were of similar magnitude for these two sets of cells.

As expected, mean firing rates were similar between control and SWE groups (control, $0.24 \pm 0.02 \mathrm{~s} / \mathrm{s}, n=49 \mathrm{vs}$ SWE, $0.28 \pm$ $0.02, n=23 ; p=0.22$ ) (Fig. $7 A$ ). We also observed no change in trial-to-trial reliability between groups (control, $0.19 \pm 0.04, n=$ 49 vs SWE, $0.23 \pm 0.02, n=23 ; p=0.18$ ), confirming the notion that firing rate potentiation arises predominantly through increased reliability. Among these neurons with the lowest firing rates, however, SWE was associated with a $3 \mathrm{~ms}$ reduction in mean onset latency (control, $25.0 \pm 0.9 \mathrm{~ms}, n=49$ vs SWE, $21.9 \pm 0.8 \mathrm{~ms}, n=23 ; p=0.026$ ) (Fig. $7 C, D$ ). Additionally, trial-to-trial spike times were significantly more precise (control, $9.67 \pm 0.54 \mathrm{~ms}, n=49$ vs SWE, $7.21 \pm 0.95 \mathrm{~ms}, n=23 ; p=$ 0.026 ) (Fig. $7 E, F$ ). The presence of altered timing and precision among SWE neurons firing at "control" rates suggests that, although enhanced temporal precision and increased firing rates 
can co-occur in the same cell, these two phenomena may arise independently.

A similar analysis was performed to dissociate the effects of reduced latency from increased firing output (supplemental Fig. 3, available at www.jneurosci.org as supplemental material). In this case, for cells exhibiting short-latency (10-20 ms) spikes in both control and SWE animals, both mean firing rate and trial-to-trial reliability were significantly increased. Thus, firing rate potentiation, reduced spike latency, and increased spike precision may be modulated independently by altered sensory experience.

\section{Plasticity of spike timing and precision in adolescent mice}

Experience-dependent plasticity in young postnatal animals generally occurs more rapidly than in adolescent or adult animals, although increasing evidence suggests that the adult neocortex retains a strong capacity for change (Glazewski and Fox, 1996; He et al., 2006; Iny et al., 2006). We performed additional experiments and also reanalyzed data collected in a previous study (Glazewski et al., 2007) to determine whether SWE could induce similar changes in spike-timing precision and reliability in adolescent animals. A comparison of evoked firing between young postnatal (P13-P16) and adolescent mice (5-8 weeks) shows increases in both layer 4 and layer 2/3. However, $24 \mathrm{~h}$ of SWE was not sufficient to induce significant changes in mean stimulus-evoked firing for layer $2 / 3$ neurons in adolescent animals (control, $1.81 \pm 0.11 \mathrm{~s} / \mathrm{s}, n=112$ cells vs SWE, $1.50 \pm 0.14, n=46$ cells; $p=$ 0.07) (supplemental Fig. 4, available at www.jneurosci.org as supplemental material). Interestingly, this deprivation did result in significant depression of responses in layer 4 (control, $2.78 \pm 0.20 \mathrm{~s} / \mathrm{s}$, $n=36$ cells vs SWE, $1.98 \pm 0.31, n=18$ cells; $p=0.028)$. Seven days of SWE was sufficient to induce potentiation of stimulusevoked firing in layer $2 / 3$ neurons $(2.55 \pm 0.19 ; n=39$ cells; $p=$ 0.00074).

We then analyzed these datasets to determine whether $7 \mathrm{~d}$ of SWE-induced changes in reliability, timing, and precision of whisker-evoked responses in adolescent animals (Fig. 8). Consistent with what was observed in young postnatal animals, $7 \mathrm{~d}$ of SWE led to a significant increase in layer $2 / 3$ response reliability compared with age-matched controls (trial-to-trial reliability, control, $0.73 \pm 0.02, n=112$ cells vs $\mathrm{SWE}, 0.86 \pm 0.02, n=39$ cells; $p=0.0019$ ) (Fig. 8A), which was not observed in layer 4 . Similarly, both the latency (control, $13.75 \pm 0.53 \mathrm{~ms}, n=112$ cells vs SWE, $9.23 \pm 0.50, n=39$ cells; $p<10^{-5}$ ) (Fig. $8 B$ ) and SD of the first evoked spike (control, $6.59 \pm 0.4 \mathrm{~ms}, n=112$ cells vs SWE, $4.49 \pm 0.54, n=39$ cells; $p=0.0083$ ) were significantly reduced by SWE in layer $2 / 3$ (Fig. $8 C$ ). These results suggest that experience-dependent plasticity in adolescent animals induces
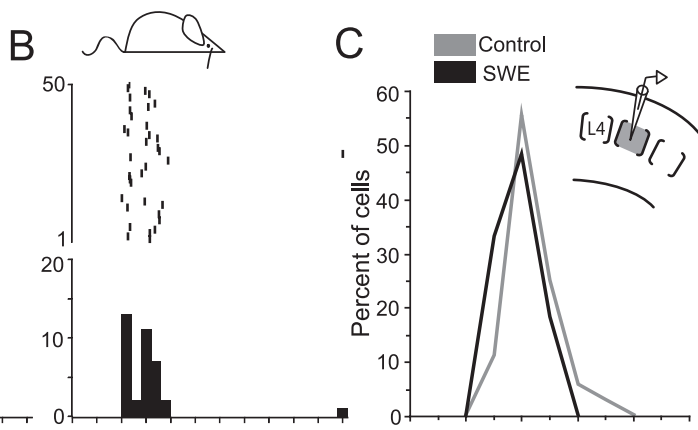

4. SWE drives earlier whisker-evoked responses in superficial layers. $A, B$, Raster and PSTH plots of layer 4 (top) and laye eflection. For trials in which more than one spike was evoked, only the first is included. C, Population histograms of onset latencies ayer 4 (top) and 2/3 (bottom) cells from control (gray; $n=36$ layer 4 cells, 76 layer 2/3 cells) and SWE (black; $n=33$ layer 4 (black) animals. ${ }^{*} p<10^{-4}$. $p$ Box .D. Mean \pm SEM onset latency for layer 4 and $2 / 3$ cells from control (gray) and SWE square represents the mean, horizontal line represents the median, top and bottom box boundaries represent the 75 th and 25 th percentiles, and vertical capped lines indicate the 95 th and 5 th percentiles. $F$, Relationship between reliability and onset latency for each layer 2/3 cell from control (gray, left) and SWE (black, right) animals. Lines show the linear regression for control (Spearman's correlation coefficient, $\rho=-0.27, p<0.05)$ and $\operatorname{SWE}(\rho=-0.42, p<0.001)$.

the same types of changes in response reliability and spike-timing precision as observed during the earlier postnatal maturation of somatosensory cortex, although these changes require longer periods of patterned sensory input (i.e., single-whisker experience) to become apparent.

\section{Discussion}

The unreliability of neocortical neurons has been widely appreciated ever since the responses of single neurons were first studied more than four decades ago. The way that the nervous system takes advantage of or, alternatively, overcomes this unreliability is an important topic for neuroscientists interested in coding (Bialek and Rieke, 1992; Mainen and Sejnowski, 1995; de Ruyter van Steveninck et al., 1997; DeWeese et al., 2005; VanRullen et al., 2005; Ermentrout et al., 2008; Tiesinga et al., 2008). However, few studies have examined whether this apparently characteristic feature of nervous system function can be modified by experience and plasticity. The data presented here indicate that unreliability 

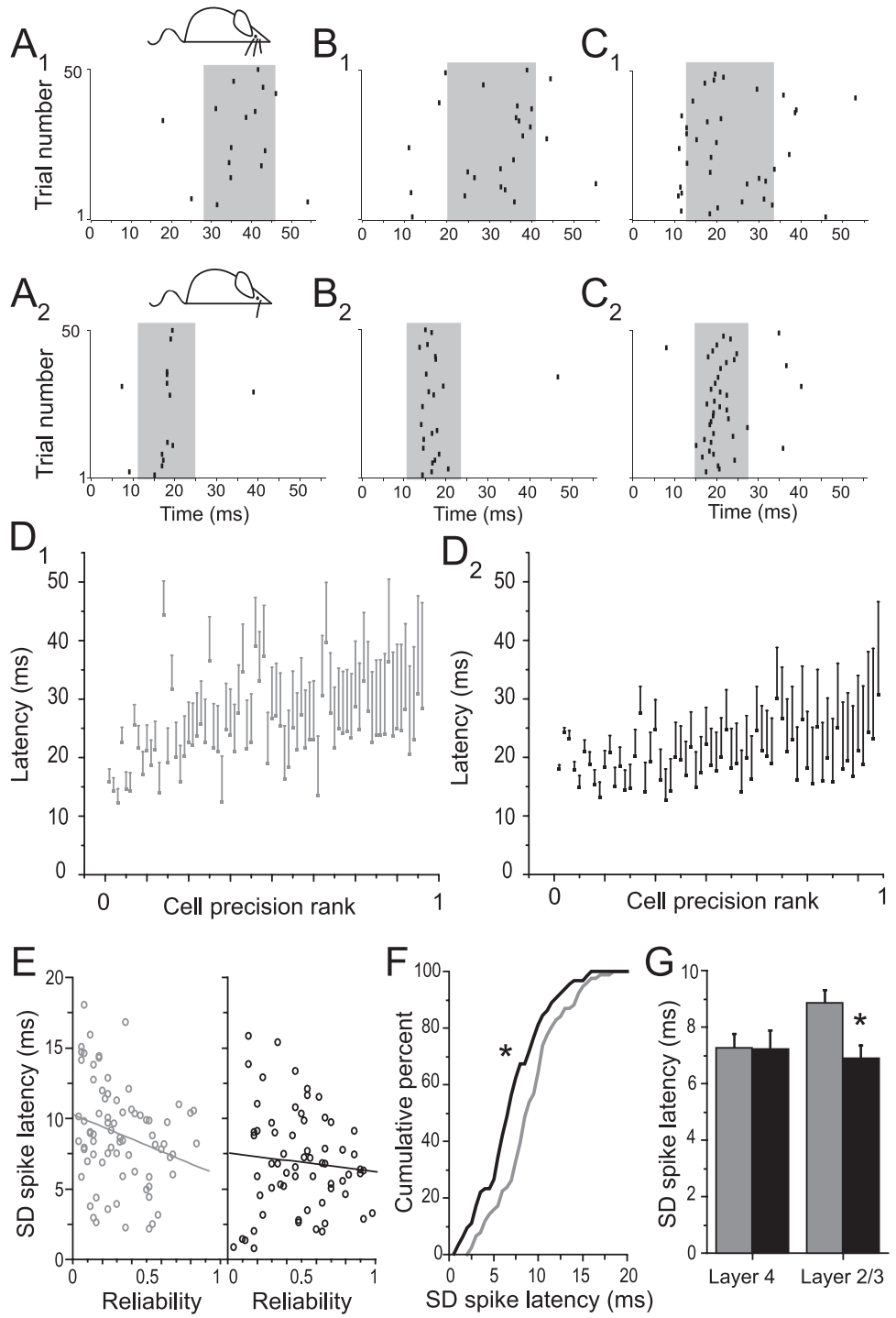

Figure 5. SWE enhances temporal precision of sensory-evoked spikes. $\boldsymbol{A}-\boldsymbol{C}$, Evoked responses in layer $2 / 3$ are more precise after SWE. $\boldsymbol{A}$, First spike raster plots for unreliable cells from control $\left(\boldsymbol{A}_{\boldsymbol{1}}\right.$; reliability of 0.32$)$ and SWE $\left(\boldsymbol{A}_{2}\right.$; reliability of 0.30$)$ animals. The highlighted region in gray is centered on the mean latency of the cell and extends \pm 1 SD. $\boldsymbol{B}$, Raster plots for reliable cells from control ( $\boldsymbol{B}_{\boldsymbol{1}}$; reliability of 0.46$)$ and SWE ( $\boldsymbol{B}_{2}$; reliability of 0.46$)$. $\boldsymbol{C}$, Raster plots for very reliable cells from control $\left(\boldsymbol{C}_{\boldsymbol{1}}\right.$; reliability of 0.72 ) and SWE ( $\boldsymbol{C}_{2} ;$ reliability of 0.82$)$. $\boldsymbol{D}$, Latency and precision of all layer $2 / 3$ cells from control $\left(\boldsymbol{D}_{1}\right)$ and SWE $\left(\boldsymbol{D}_{2}\right)$ animals. Cells are ranked according to the $S D$ of their response, such that the most precise cells are shown to the left and least precise cells to the right. Square symbols indicate the mean onset latency; lines indicate 1 SD from the mean (for clarity only, the positive direction is shown). $\boldsymbol{E}$, Correlation between spike precision and reliability for control (left, gray) and SWE (right, black) cells. Lines show the linear regression for control (Spearman's correlation coefficient, $\rho=-0.25, p<0.05)$ and SWE $(\rho=-0.05, p=0.69)$. $\boldsymbol{F}$, Cumulative distribution of layer 2/3 spike precision for cells from control (gray) and SWE (black) mice. ${ }^{*} p<0.001$. G, Mean \pm SEM layer 4 and layer $2 / 3$ spike precision for cells from control and SWE. ${ }^{*} p<0.005$.

in the presence and timing of stimulus-evoked spikes is not a fixed parameter in neocortical circuits but can be enhanced during the course of experience-dependent plasticity. The ability to modulate spike timing and precision in neocortical circuits demonstrated here will provide an experimental substrate to constrain specific hypotheses about how timing and precision influence neural coding strategies.

Here we evaluate the manner by which stimulus-evoked firing in layer $2 / 3$ neurons is enhanced during experience-dependent plasticity in the barrel cortex. We find that this enhancement is attributable in part to a pronounced reduction of stimulus trials in which no firing is elicited (a reduction in failure rate) and is also correlated with a significant increase in spike-timing preci- sion. Evoked spikes are more reliable across stimulus trials, come earlier after the stimulus, and show less trial-to-trial variability in response times. Although these changes can occur rapidly in young postnatal animals, we could also observe them in adolescent animals with a longer duration of single-whisker experience.

\section{Mechanisms to enhance spike-timing precision and response reliability}

What are the cellular and synaptic mechanisms that might underlie increased response reliability and timing precision? Our previous results indicate that excitatory layer 4 to layer $2 / 3$ synapses undergo synaptic strengthening after SWE in young postnatal animals (Clem and Barth, 2006), and it is reasonable to expect that a larger excitatory synaptic drive might underlie the increase in reliability as well as reduced onset latency observed here. Interestingly, Daw et al. (2006) have observed that EPSPs become more tightly coupled to spikes after long-term potentiation at thalamocortical synapses in acute brain slices from early postnatal animals. Because it has been shown that strengthening of excitatory layer 4 to layer $2 / 3$ synapses occurs during SWE (Clem and Barth, 2006; Clem et al., 2008), it is reasonable to expect that enhanced EPSPspike coupling may be occurring here. An increase in the amplitude or change in the kinetics of the EPSP response [perhaps attributable to the trafficking of fast-decay, calcium-permeable AMPA receptors (Clem and Barth, 2006; Plant et al., 2006)] would enhance reliability and timing precision in the neocortex.

Activity-dependent changes in voltagegated or leak potassium conductances [such as a reduction in A-type current (Bernard et al., 2004)] or a reduction in AP threshold could also reduce the latency of the first stimulus-driven spike. However, our own experimental data do not suggest that AP threshold is hyperpolarized after SWE (Barth et al., 2004). Because our analysis suggests that reduced spike latencies and enhanced spike-timing precision may occur independently of firing rate potentiation, it is possible that SWEinduced changes in synaptic input may work in concert with altered intrinsic excitability of the cell. The relative timing and contribution of these effects remains unclear.

\section{Precisely timed and reliable spikes occur at shorter latencies}

The timing of the first spike after a stimulus has been proposed to carry the most information compared with later spikes or overall spike rates (Panzeri et al., 2001; Johansson and Birznieks, 2004; Saal et al., 2009). The analysis presented here was focused on the first spike after the stimulus, in part because most neurons did not show more than a single AP in a given stimulus trial $(<8 \%$ of 
stimulus trials in control animals showed more than one spike in the first $50 \mathrm{~ms}$ ). Our observation that the spike output of an individual neuron was typically below $1 \mathrm{~s} / \mathrm{s}$, even over a time window of several hundred milliseconds, is consistent with the conclusions that analysis of "spike rates" for individual neurons may be an inappropriate measure of information transmission in the barrel cortex.

The observation that layer $2 / 3$ neurons with the shortest-latency responses exhibited the most reliable firing suggests that changes in the direct response to layer 4 activation may be directly related to the improvements in spike timing and reliability observed here. Because firing rates in layer 4 typically exceed those observed in layer $2 / 3$ neurons in control, undeprived animals, it has been proposed that layer $2 / 3$ acts as a gate for incoming sensory information (Feldmeyer et al., 2002; Sarid et al., 2007). After SWE, we find that firing rates in layer 4 and layer $2 / 3$ are not significantly different, suggesting that the gating function of layer $2 / 3$ may be reduced. Because there is direct experimental evidence that excitatory synapses between layer 4 and layer 2/3 neurons are strengthened by SWE (Clem and Barth, 2006) and because early spikes are required to generate later network activity, improved coupling at these synapses will disproportionately influence improvements in timing and reliability at both early and later time bins.

\section{Enhanced timing and reliability persist in adult plasticity}

The most rapid and pronounced effects on spike latency, timing precision, and reliability observed were in young postnatal animals aged $\mathrm{P} 13-\mathrm{P} 16$, a period that coincides with the rapid maturation of layer $2 / 3$ circuits (Maravall et al., 2004). Because these changes could also be induced in young adult animals after longer periods of SWE, a discrete critical period for experience-dependent improvements in timing precision and reliability is unlikely. Rather, we propose that, given the right conditions, experience-dependent enhancement in timing and reliability of neocortical neuronal firing may be engaged across the lifespan of the animal.

It is worth noting that we found that short periods of SWE $(24 \mathrm{~h})$ in adolescent mice had opposite effects on firing rate potentiation, latency, and timing precision than the same duration of SWE in young postnatal animals. Indeed, other investigations have observed that response latency is increased after short durations of whisker-pairing-induced response potentiation in young adult animals (Armstrong-James et al., 1994). In adolescent mice, we observed that spared-whisker-evoked responses were depressed in the cognate barrel column (particularly in layer 4), latencies went up (not down), and timing precision was degraded after $24 \mathrm{~h}$ of SWE. The explanation for these phenomena is unclear but may be related to rapid enhancement of inhibition that has been observed under some whisker-stimulation conditions (Knott et al., 2002). For example, because inhibition that is tightly coupled to excitation would act to shunt depolarizing EPSPs, it could easily reduce reliability of stimulus-evoked responses.

\section{Latency changes during sensory deprivation}

A review of the literature is remarkable in that it reveals few studies that have examined or reported how firing latency and precision are altered during sensory-induced response potentiation. Generally, the cortical effects of sensory deprivation have been better characterized than sensory-induced response potentiation, the subject of the current study. For example, partial anesthesia of the facial nerve blocks short-latency responses in the thalamus (Nicolelis et al., 1993), although neocortical responses were not evaluated in this study. Acute whisker clipping reduces the latency of surround whisker responses in layer $2 / 3$, in a manner consistent with induction of spike-timing-dependent depression from layer 4 to layer $2 / 3$ synapses in the deprived barrel column (Celikel et al., 2004). Although the study by Celikel et al. was focused on the immediate effects of whisker inactivity, we predict that this decrease in latency of surround (spared) whisker 

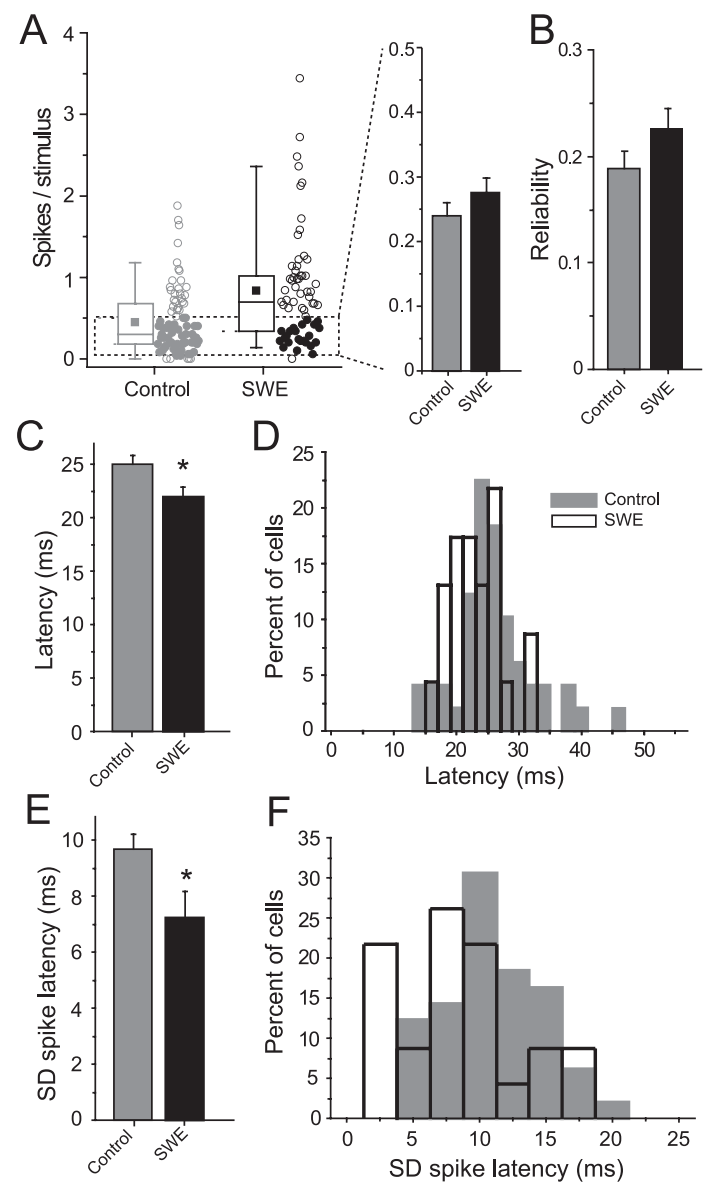

Figure 7. Experience-dependent enhancement of temporal precision is independent of firing rate potentiation. $A$, Selection of neurons from control and SWE with similar firing rates. Left, Box and scatter plots of response magnitudes for all recorded layer $2 / 3$ neurons. Neurons firing in the range $>0$ to $\leq 0.5 \mathrm{~s} / \mathrm{s}$ (filled gray and black circles, outlined region on left) were selected for additional analysis. Right, Mean \pm SEM response magnitudes for selected neurons from control $(n=49)$ and SWE $(n=23 ; p>0.1) . B$, Mean \pm SEM response reliability ( $p>$ $0.1) . C, D$, Mean \pm SEM layer $2 / 3$ onset latency and latency histograms for neurons from control (filled gray bars) and SWE (open black bars). $E, F$, Mean \pm SEM layer $2 / 3$ response precision (left) and precision histograms (right). ${ }^{*} p<0.05$.

responses would likely to persist over time. In our experimental setup, we would predict that spiking responses to the single spared whisker would show reduced latency in surrounding, sensory-deprived barrel columns during longer periods of sensory deprivation. However, it is worth noting that surround responses in very young animals are negligible and that evoking any APs by stimulation of a nonprincipal whisker rarely, if ever, occurs. Thus, it is likely that we would not be able to detect any latency changes in deprived barrel columns in the young postnatal animals used in this study.

Although the form of whisker activity used to induce response potentiation (SWE) in this study is not likely to occur in the wild, whisker trimming between conspecifics has been described in cage-raised animals (Strozik and Festing, 1981) and is present in our own mouse colony. We note that the SWE preparation was developed as a way in which to better understand whether, and by what cellular and molecular mechanisms, spike reliability, timing precision, and response latency could be enhanced during experience-dependent plasticity. It will be of interest to examine whether these features are altered in other experimental paradigms, such as naturalistic, sensory-enriched housing conditions,
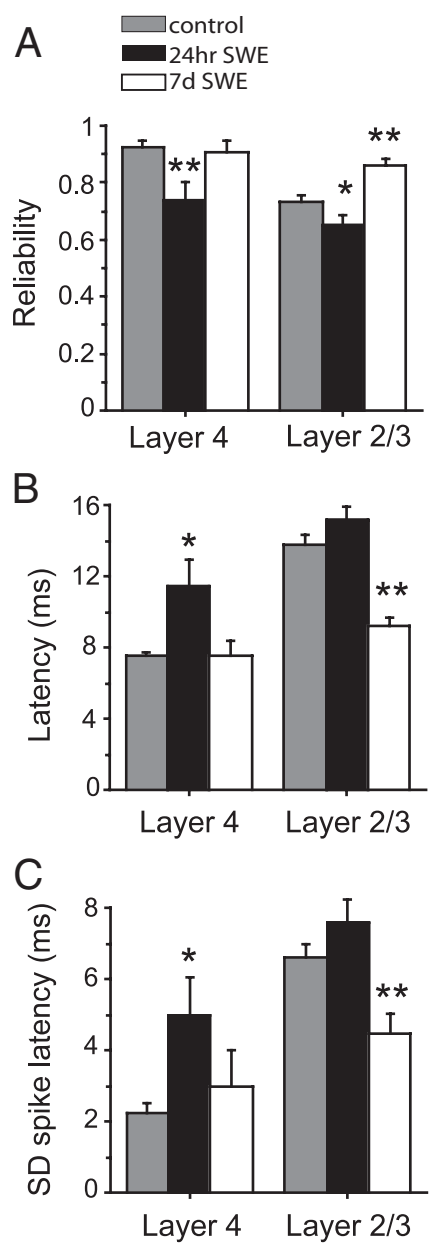

Figure 8. Experience-dependent changes in reliability and spike-timing precision in adolescent mice. $\boldsymbol{A}$, Mean \pm SEM response reliability calculated for layer 4 and layer $2 / 3$ cells from control (gray; $n=36$ layer 4 cells, 112 layer $2 / 3$ cells), 24 h SWE (black; $n=18$ for layer 4,46 for layer 2/3), and $7 \mathrm{dSWE}$ (white; $n=11$ for layer 4,39 for layer 2/3) mice. ${ }^{*} p<0.05$; $^{* *} p<0.01$. $\boldsymbol{B}$, Mean \pm SEM response onset latency for the same groups as in $\boldsymbol{A}$. ${ }^{*} p<0.05 ;{ }^{* *} p<10^{-4}$. C, Mean \pm SEM response precision. ${ }^{*} p<0.05$; $^{* *} p<0.01$.

that alter receptive field properties and firing output in somatosensory cortex (Polley et al., 1999, 2004).

\section{Potentiation of recurrent activity in the cortical column}

Enhanced trial-to-trial reliability and spike-timing precision is expected to enhance temporal summation of EPSPs in postsynaptic neurons; at a population level, this is likely to enhance recurrent activity in layer $2 / 3$ as well as other interconnected areas within the cortical column (specifically layer 5). In fact, we do observe a significant increase in firing at longer latencies (55-205 $\mathrm{ms}$ after the stimulus) in SWE-treated animals. Because there was no increase in evoked or spontaneous firing activity for layer 4 neurons or in spontaneous firing for layer $2 / 3$ neurons, this stimulus-associated activity is likely to be attributable to more efficient transmission of stimulus information into and propagation through the layer 2/3 network. Although this may be attributable in part to increases in the strength of excitatory synapses and EPSP-spike coupling, reduced inhibition in local supragranular and infragranular circuits might also contribute to the extended duration of stimulus-evoked firing. Other slow conductances, such as NMDA receptor (NMDAR)-mediated EPSCs, might provide a source of depolarization that could contribute to later APs (Armstrong-James et al., 1993). Indeed, potential in- 
creases in NMDAR EPSCs, by either direct potentiation of these currents or the addition of NMDAR-only (silent) synapses, might have a profound influence on long-latency responses.

Potentiation of stimulus-evoked firing in the barrel cortexand corresponding changes in spike timing and reliability-have not been well correlated with behavioral improvements in perceptual ability, and so questions about how these changes can impact sensory detection present an exciting avenue for additional investigation. Previous studies in invertebrates have observed that improved timing and reliability can occur during olfactory learning (Stopfer and Laurent, 1999; Bazhenov et al., 2005), although these studies were performed over significantly shorter timescales (hours) that what is described here. Nonetheless, the convergence of these results provides good experimental data that show that improved timing and reliability may be a common strategy for enhancing neuronal responsiveness, reducing detection thresholds for weak stimuli, and improving information transmission across neural networks.

\section{References}

Arabzadeh E, Zorzin E, Diamond ME (2005) Neuronal encoding of texture in the whisker sensory pathway. PLoS Biol 3:e17.

Armstrong-James M, Welker E, Callahan CA (1993) The contribution of NMDA and non-NMDA receptors to fast and slow transmission of sensory information in the rat SI barrel cortex. J Neurosci 13:2149-2160.

Armstrong-James M, Diamond ME, Ebner FF (1994) An innocuous bias in whisker use in adult rats modifies receptive fields of barrel cortex neurons. J Neurosci 14:6978-6991.

Barth AL, McKenna M, Glazewski S, Hill P, Impey S, Storm D, Fox K (2000) Upregulation of cAMP response element-mediated gene expression during experience-dependent plasticity in adult neocortex. J Neurosci 20:4206-4216.

Barth AL, Gerkin RC, Dean KL (2004) Alteration of neuronal firing properties after in vivo experience in a FosGFP transgenic mouse. J Neurosci 24:6466-6475.

Bazhenov M, Stopfer M, Sejnowski TJ, Laurent G (2005) Fast odor learning improves reliability of odor responses in the locust antennal lobe. Neuron 46:483-492.

Bernard C, Anderson A, Becker A, Poolos NP, Beck H, Johnston D (2004) Acquired dendritic channelopathy in temporal lobe epilepsy. Science 305:532-535.

Bialek W, Rieke F (1992) Reliability and information transmission in spiking neurons. Trends Neurosci 15:428-434.

Brecht M, Sakmann B (2002) Whisker maps of neuronal subclasses of the rat ventral posterior medial thalamus, identified by whole-cell voltage recording and morphological reconstruction. J Physiol 538:495-515.

Bruno RM, Sakmann B (2006) Cortex is driven by weak but synchronously active thalamocortical synapses. Science 312:1622-1627.

Celikel T, Szostak VA, Feldman DE (2004) Modulation of spike timing by sensory deprivation during induction of cortical map plasticity. Nat Neurosci 7:534-541.

Cheetham CE, Hammond MS, Edwards CE, Finnerty GT (2007) Sensory experience alters cortical connectivity and synaptic function site specifically. J Neurosci 27:3456-3465.

Clem RL, Barth A (2006) Pathway-specific trafficking of native AMPARs by in vivo experience. Neuron 49:663-670.

Clem RL, Celikel T, Barth AL (2008) Ongoing in vivo experience triggers synaptic metaplasticity in the neocortex. Science 319:101-104.

Daw MI, Bannister NV, Isaac JT (2006) Rapid, activity-dependent plasticity in timing precision in neonatal barrel cortex. J Neurosci 26:4178-4187.

de Kock CP, Bruno RM, Spors H, Sakmann B (2007) Layer- and cell-typespecific suprathreshold stimulus representation in rat primary somatosensory cortex. J Physiol 581:139-154.

de Ruyter van Steveninck RR, Lewen GD, Strong SP, Koberle R, Bialek W (1997) Reproducibility and variability in neural spike trains. Science 275:1805-1808.

DeWeese MR, Hromádka T, Zador AM (2005) Reliability and representational bandwidth in the auditory cortex. Neuron 48:479-488.

Diamond ME, Huang W, Ebner FF (1994) Laminar comparison of somatosensory cortical plasticity. Science 265:1885-1888.
Ermentrout GB, Galán RF, Urban NN (2008) Reliability, synchrony and noise. Trends Neurosci 31:428-434.

Feldmeyer D, Lübke J, Silver RA, Sakmann B (2002) Synaptic connections between layer 4 spiny neurone-layer $2 / 3$ pyramidal cell pairs in juvenile rat barrel cortex: physiology and anatomy of interlaminar signalling within a cortical column. J Physiol 538:803-822.

Feldmeyer D, Lübke J, Sakmann B (2006) Efficacy and connectivity of intracolumnar pairs of layer $2 / 3$ pyramidal cells in the barrel cortex of juvenile rats. J Physiol 575:583-602.

Glazewski S, Fox K (1996) Time course of experience-dependent synaptic potentiation and depression in barrel cortex of adolescent rats. J Neurophysiol 75:1714-1729.

Glazewski S, McKenna M, Jacquin M, Fox K (1998) Experience-dependent depression of vibrissae responses in adolescent rat barrel cortex. Eur J Neurosci 10:2107-2116.

Glazewski S, Benedetti BL, Barth AL (2007) Ipsilateral whiskers suppress experience-dependent plasticity in the barrel cortex. J Neurosci 27: 3910-3920.

He HY, Hodos W, Quinlan EM (2006) Visual deprivation reactivates rapid ocular dominance plasticity in adult visual cortex. J Neurosci 26: 2951-2955.

Houweling AR, Brecht M (2008) Behavioural report of single neuron stimulation in somatosensory cortex. Nature 451:65-68.

Huber D, Petreanu L, Ghitani N, Ranade S, Hromádka T, Mainen Z, Svoboda K (2008) Sparse optical microstimulation in barrel cortex drives learned behaviour in freely moving mice. Nature 451:61-64.

Iny K, Heynen AJ, Sklar E, Bear MF (2006) Bidirectional modifications of visual acuity induced by monocular deprivation in juvenile and adult rats. J Neurosci 26:7368-7374.

Johansson RS, Birznieks I (2004) First spikes in ensembles of human tactile afferents code complex spatial fingertip events. Nat Neurosci 7:170-177.

Jones LM, Depireux DA, Simons DJ, Keller A (2004) Robust temporal coding in the trigeminal system. Science 304:1986-1989.

Kara P, Reinagel P, Reid RC (2000) Low response variability in simultaneously recorded retinal, thalamic, and cortical neurons. Neuron 27:635-646.

Kerr JN, de Kock CP, Greenberg DS, Bruno RM, Sakmann B, Helmchen F (2007) Spatial organization of neuronal population responses in layer 2/3 of rat barrel cortex. J Neurosci 27:13316-13328.

Knott GW, Quairiaux C, Genoud C, Welker E (2002) Formation of dendritic spines with GABAergic synapses induced by whisker stimulation in adult mice. Neuron 34:265-273.

Lefort S, Tomm C, Floyd Sarria JC, Petersen CC (2009) The excitatory neuronal network of the $\mathrm{C} 2$ barrel column in mouse primary somatosensory cortex. Neuron 61:301-316.

Ma WJ, Beck JM, Latham PE, Pouget A (2006) Bayesian inference with probabilistic population codes. Nat Neurosci 9:1432-1438.

Mainen ZF, Sejnowski TJ (1995) Reliability of spike timing in neocortical neurons. Science 268:1503-1506.

Maravall M, Stern EA, Svoboda K (2004) Development of intrinsic properties and excitability of layer $2 / 3$ pyramidal neurons during a critical period for sensory maps in rat barrel cortex. J Neurophysiol 92:144-156.

Nicolelis MA, Lin RC, Woodward DJ, Chapin JK (1993) Induction of immediate spatiotemporal changes in thalamic networks by peripheral block of ascending cutaneous information. Nature 361:533-536.

Oviedo H, Reyes AD (2002) Boosting of neuronal firing evoked with asynchronous and synchronous inputs to the dendrite. Nat Neurosci 5:261-266

Panzeri S, Petersen RS, Schultz SR, Lebedev M, Diamond ME (2001) The role of spike timing in the coding of stimulus location in rat somatosensory cortex. Neuron 29:769-777.

Plant K, Pelkey KA, Bortolotto ZA, Morita D, Terashima A, McBain CJ, Collingridge GL, Isaac JT (2006) Transient incorporation of native GluR2-lacking AMPA receptors during hippocampal long-term potentiation. Nat Neurosci 9:602-604.

Polley DB, Chen-Bee CH, Frostig RD (1999) Two directions of plasticity in the sensory-deprived adult cortex. Neuron 24:623-637.

Polley DB, Kvasnák E, Frostig RD (2004) Naturalistic experience transforms sensory maps in the adult cortex of caged animals. Nature 429:67-71.

Reinagel P, Reid RC (2000) Temporal coding of visual information in the thalamus. J Neurosci 20:5392-5400. 
Reinagel P, Reid RC (2002) Precise firing events are conserved across neurons. J Neurosci 22:6837-6841.

Saal HP, Vijayakumar S, Johansson RS (2009) Information about complex fingertip parameters in individual human tactile afferent neurons. J Neurosci 29:8022-8031.

Sarid L, Bruno R, Sakmann B, Segev I, Feldmeyer D (2007) Modeling a layer 4-to-layer 2/3 module of a single column in rat neocortex: interweaving in vitro and in vivo experimental observations. Proc Natl Acad Sci U S A 104:16353-16358.

Shadlen MN, Newsome WT (1998) The variable discharge of cortical neurons: implications for connectivity, computation, and information coding. J Neurosci 18:3870-3896.
Stern EA, Maravall M, Svoboda K (2001) Rapid development and plasticity of layer 2/3 maps in rat barrel cortex in vivo. Neuron 31:305-315.

Stopfer M, Laurent G (1999) Short-term memory in olfactory network dynamics. Nature 402:664-668.

Strozik E, Festing MF (1981) Whisker trimming in mice. Lab Anim 15:309-312.

Takahashi T, Svoboda K, Malinow R (2003) Experience strengthening transmission by driving AMPA receptors into synapses. Science 299:1585-1588.

Tiesinga P, Fellous JM, Sejnowski TJ (2008) Regulation of spike timing in visual cortical circuits. Nat Rev Neurosci 9:97-107.

VanRullen R, Guyonneau R, Thorpe SJ (2005) Spike times make sense. Trends Neurosci 28:1-4. 